\title{
Trennung der alkalischen Phosphatase Isoenzyme mit Lektin-Affinitäts-Elektrophorese und Lektin-Fällung
}

\author{
Separation of Alkaline Phosphatase Isoenzymes by Lectin Affinity Electrophoresis and \\ Lectin Precipitation
}

H.-W. Schiwara

Labor Schiwara, von Winterfeld, Pfanzelt, Bremen

\begin{abstract}
Zusammenfassung:
Die Isoenzyme der alkalischen Phosphatase werden elektrophoretisch in dünnschichtigen lektinhaltigen und lektinfreien Agarosegelen getrennt. Mit der Kombination dieser beiden Elektrophoresetechniken können alle Isoenzyme der alkalischen Phosphatase (Leber-, Gallengangs-, Knochen-, Placènta-, Dünndarm- und Makro$A P)$ zuverlässig identifiziert und quantitativ bestimmt werden. Die elektrophoretisch gemessenen Aktivitäten des Knochen-Isoenzyms korrelieren gut mit den Isoenzym-Aktivitäten, die mit den Lektin-Fällungs-Methoden von Rosalki und Boehringer Mannheim ermittelt wurden. Allerdings finden sich besonders mit der Methode von Rosalki, aber auch mit dem Fällungsreagenz von Boehringer Mannheim in einer relativ großen Zahl von Seren falsch erhöhte Aktivitäten der Knochen-AP. Seltener vorkommende Isoenzyme wie Dünndarm-, Placenta- und Makro-AP können mit den Lektin-Fällungs-Methoden als Leber-Isoenzym der alkalischen Phosphatase fehlgedeutet werden. Die Lektin-Fällungs-Methoden könnten sich wegen ihrer guten Reproduzierbarkeit für die Messung der Knochen-AP bei Verlaufskontrollen eignen. Das Isoenzym-Muster der alkalischen Phosphatase sollte aber wenigstens einmal mit einem qualifizierten elektrophoretischen Verfahren bestimmt werden.
\end{abstract}

Schlüsselwörter:

Alkalische Phosphatase Isoenzyme - Lektin-Affinitäts-Elektrophorese - Lektin-Fällung

\begin{abstract}
Summary:
The isoenzymes of alkaline phosphatase are separated electrophoretically in lectin-containing and lectin-free thinlayer agarose gels. Combining these two electophoretical techniques liver, biliary, bone, placental, intestinal and makro isoenzymes of alkaline phosphatase can be identified and quantified reliably. The electrophoretically analyzed activities of the bone isoenzyme correlate well with the activities measured by the lectin precipitation methods of Rosalki and Boehringer Mannheim. However, in many sera falsely elevated activities of bone alkaline phosphatase are found, especially by the method of Rosalki but also by the method of Boehringer Mannheim. Using lectin precipitation methods, rare intestinal, placental and makro alkaline phosphatases cannot be identified but can be misinterpreted as liver alkaline phosphatase. Lectin precipitation methods can be useful in follow up studies of bone alkaline phosphatase because of their good reproducibility. In this case the isoenzyme pattern should be investigated at least once by a reliable electrophoretic technique.
\end{abstract}

Keywords:

Alkaline phosphatase isoenzymes - lectin affinity electrophoresis - lectin precipitation

\section{Einleitung}

Die Trènnung der Isoenzyme der alkalischen Phosphatase ist zur Abklärung einer erhöhten Aktivität der alkalischen Phosphatase im Serum diagnostisch interessant, stellt jedoch ein erhebliches analytisches Problem dar. In einer Übersichtsarbeit anläßlich des „80. Geburtstages” der alkalischen Phosphatase wurden zahlreiche unterschiedliche Methoden dargestellt (1). Eine interessante neuere Variante ist die Verwendung von Weizenkeimlektin, das spezifisch das Knochen-Isoenzym der alkalischen Phosphatase präzipitieren soll. Weizenkeimlektin wird sowohl als Fällungsreagenz für die quantitative Beștimmung des Knochen-Isoenzyms der alkalischen Phosphatase $(2,3)$ als auch zur Verbesserung der elektrophoretischen Trennung der Isoenzyme der alkalischen Phosphatase eingesetzt $(4,5,6)$. Hier wird über eine Lektin-Affi-

\section{-}

nitäts-Elektrophorese in Dünnschicht-Agarosegelen und den Vergleich mit zwei Lektin-Fällungs-Methoden berichtet.

\section{Material und Methoden}

Weizenkeimlektin, Trizma-Barbitalpuffer $\mathrm{pH}$ 8,9, BCIPPhosphatase-Substrat Lösung und Surfactant Solution von Sigma Chemie GmbH, Deisenhofen. Antiserum gegen Placenta und Ficin von Beckman Instruments $\mathrm{GmbH}$, München. Fällungsreagenz für humane alkalische Phosphátase aus Knochen von Boehringer Mannheim $\mathrm{GmbH}$, Mannheim. Gelbond-Filme für Agarose von Pharmacia LKB GmbH, Freiburg.

Die Bestimmung des Knochen-Isoenzyms der alkalischen Phosphatase erfolgte mit der Lektin-Fällungs-Methode 

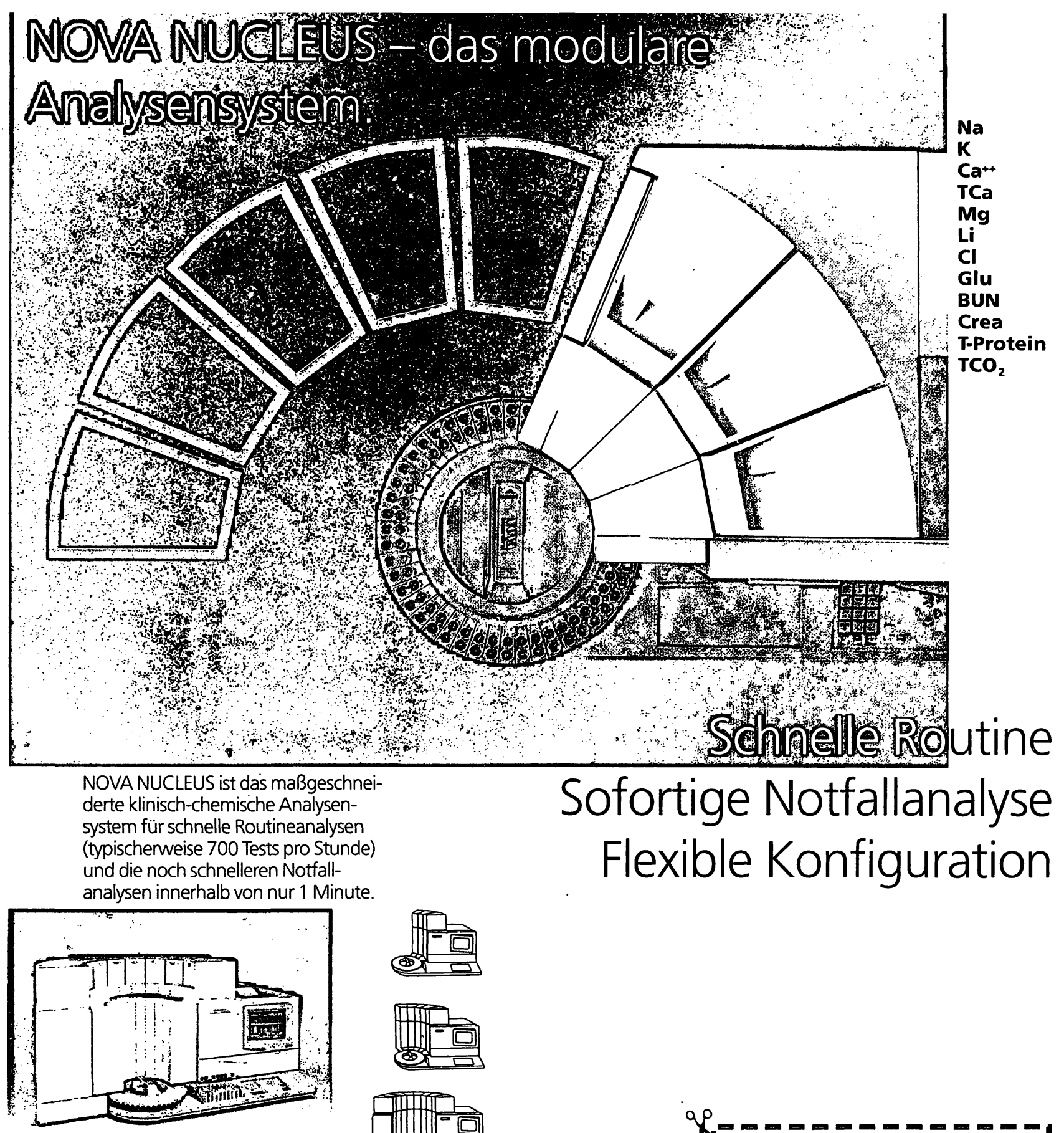

Und mit unserer neuen modularen

Bauweise können Sie sich Ihren Analysenplatz so zusammienstellen, wie Sie inn benötigen. Nicht zu klein und nicht zu groß. Ökonomisch und enweiterbar.

Gern informieren wir Sie ausführlich.
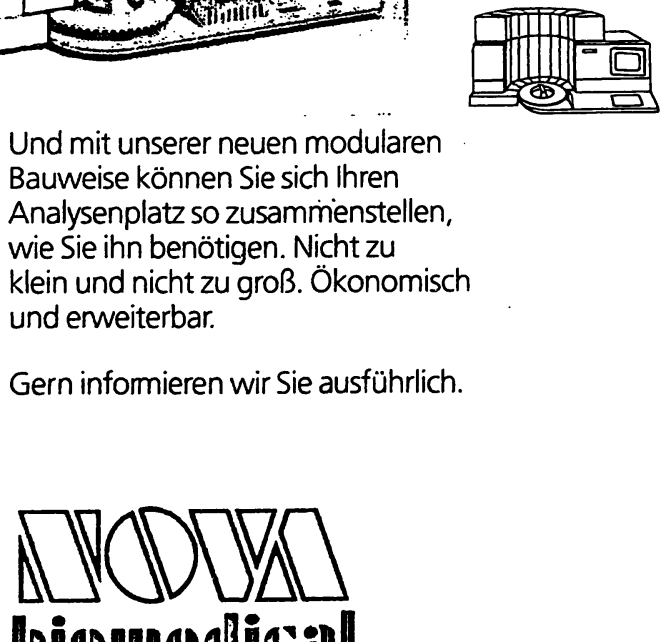

\section{boionne olic:all}

NOVA BIOMEDICAL GIMBH

Adam-Opel-Straße 19a

6074 Rödermark

Tolefon $\cap 6 \cap 74 / 5 \cap \cap>1$

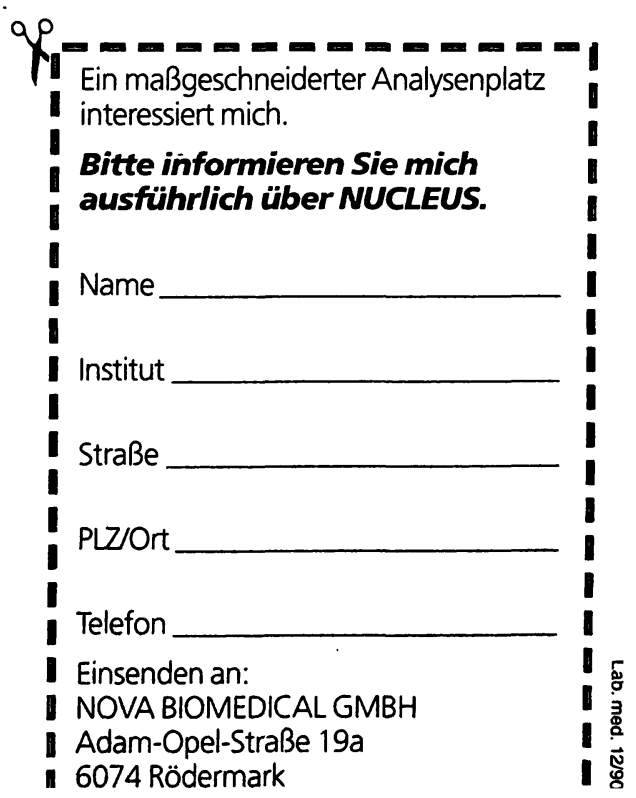




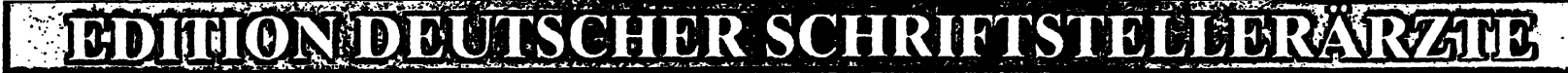

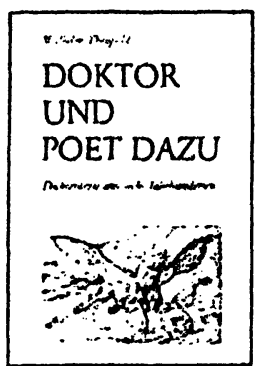

Literaturpreis 1988 der Bundesăratekammer WILHELM THEOPOLD Doktor und Poet dazu

Dichterärzte aus sechs Jahrhunderten 428 Seiten, geb. 39, 80 DM, 2. Aufl. ISBN 3-87409-032-9

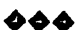

\begin{tabular}{|c|c|}
\hline \multirow{3}{*}{$\begin{array}{l}\text { Bines Menschen } \\
\text { Wege und Unwege }\end{array}$} & \\
\hline & ensc \\
\hline & $\begin{array}{l}\text { Wege und } \\
\text { Umwege }\end{array}$ \\
\hline & $\begin{array}{l}208 \text { Seiten, } \\
\text { Leineneinband, } \\
29,800 \mathrm{DM} \\
\text { ISBN 3-87409-04C }\end{array}$ \\
\hline & 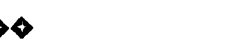 \\
\hline & $\begin{array}{l}\text { JÖRG MARTIN } \\
\text { PÖNNIGHAUS }\end{array}$ \\
\hline & $\begin{array}{l}\text { Am Bahnhof } \\
\text { und andere } \\
\text { Geschichten }\end{array}$ \\
\hline & $\begin{array}{l}144 \text { Seiten, } \\
\text { Leineneinband, } \\
24,80 \text { DM } \\
\text { ISBN 3-87409-04 }\end{array}$ \\
\hline
\end{tabular}

$-\frac{8}{6}<-$

Verlag Kirchheim, Postfach 2524, 6500 Mainz

\section{Bestellschein}

Ich bestelle folgende Titel (bitte ankreuzen):

$\square$ Doktor und Poet dazu

$39,80 \mathrm{DM}$

$\square$ Weggefährten

$\square$ Der Schwimmgürtel

$\square$ Hinter Bremsspur und Blaulicht

$\square$ Trotz Rollstuhl und Sozialdienst

$\square$ C'est la vie

口 Alfred Döblin

$\square$ Sterne über fernen Ländern

$\square$ Die Porzellanpendüle

$\square$ Der Schneemann

$\square$ vorwiegend heiter

$\square$ Eines Menschen Wege und Umwege

$\square$ Am Bahnhof und andere Geschichten

$16,80 \mathrm{DM}$

$15,80 \mathrm{DM}$

$16,80 \mathrm{DM}$

$16,80 \mathrm{DM}$

$19,80 \mathrm{DM}$

$18,00 \mathrm{DM}$

$24,80 \mathrm{DM}$

$29,80 \mathrm{DM}$

$19,80 \mathrm{DM}$

$19,80 \mathrm{DM}$

$29,80 \mathrm{DM}$

$24,80 \mathrm{DM}$

\section{Name}

Straße

PLZ

Datum/Unterschrift

Lab. med. 12/90

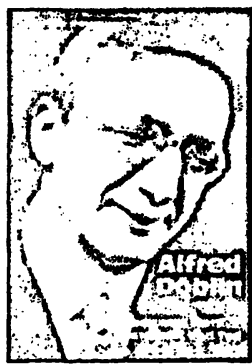

HARALD NEUMANN Alfred Döblin

Leben und Werk

Krankhelt und Tod

112 Seiten, Broschur.

$18.00 \mathrm{DM}$

ISBN 3-87409-031-0

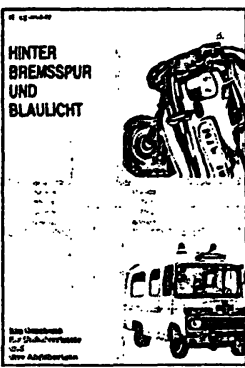

HANS SPIECKER

\section{Hinter}

Bremsspur und

Blaulicht

Ein Geschenk für

Unfallverletzte und

ihre Angehörigen

69 Seiten, 16,80 DM

ISBN 3-87409-033-7

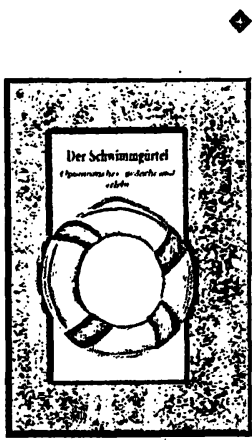

$\diamond \diamond$

HELMUT KAATZSCH

\section{Der}

Schwimmgürtel

Optimistisches, gedacht und erlebt

40 Seiten, Broschur,

15,80 DM

ISBN 3-87409-027-2

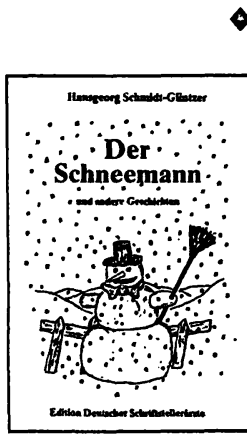

$\diamond \diamond$

HANSGEORG

SCHMIDT-GLINTZER

\section{Der}

\section{Schneemann}

und andere

Geschichten

92 Seiten.

Leineneinband,

$19,80 \mathrm{DM}$

ISBN 3-87409-037-X

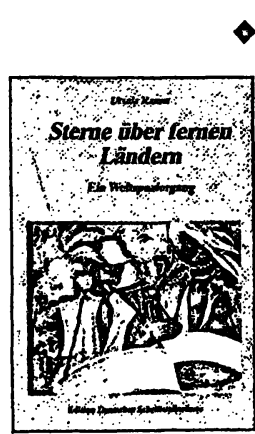

$+8$

Literaturpreis 1990 der Bundesärztekammer URSULA RAMM

Sterne über ferneń Ländern Ein Weltspaziergang 148 Seiten, Leineneinband, 24,80 DM ISBN 3-87409-035-3

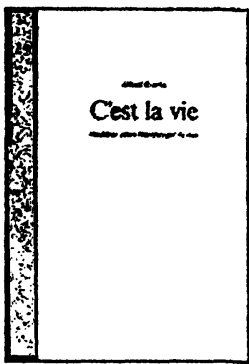

ALFRED ROTTLER

C'est la vie

Nachlese eines

Nürnberger Arztes

108 Seiten, geb.

19,80 DM

ISBN 3-87409-034-5

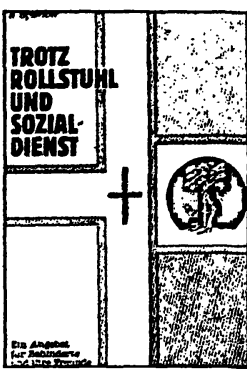

HANS SPIECKER Trotz Rollstuhl und Sozialdienst

Ein Angebot für Behinderte und ihre Freunde 64 Seiten, Broschur 16,80 DM

ISBN 3-87409-029-9

\section{$\diamond \bullet$}

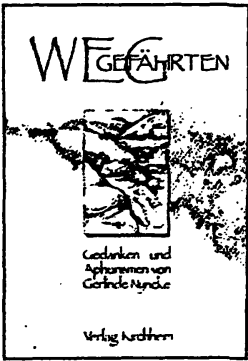

GERLINDE NYNCKE

\section{Weggefährten}

Gedanken und

Aphorismen

48 Seiten, Leineneinband, 16,80 DM, 2. Aufl.

ISBN 3-87409-043-4

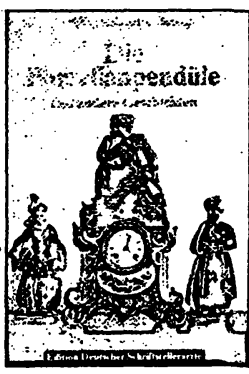

WOLFGANG LOHMANN

\section{Die Porzellan-} pendüle und andere Geschichten

224 Seiten, Leineneinband, $29,80 D M$

ISBN 3-87409-036-1

\section{$\leftrightarrow$}

vorwiegend he iter aus dem loben eines Nervonazzlos Yoo

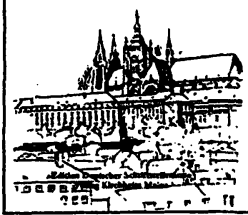

ANTON CLEMENT

vorwiegend heiter

aus dem Leben eines Nervenarztes

88 Seiten, Leineneinband, $19,80 \mathrm{DM}$

ISBN 3-87409-039-6 
von Rosalki und Ying Foo (2) sowie mit dem Fällungsreagenz von Boehringer Mannheim (7). Die Lektin-AffinitätsElektrophorese wurde nach dem Verfahren von Schreiber und Whitta (5) durchgeführt. Die Methode wurde durch Verwendung von $0,5 \mathrm{~mm}$ dünnen Agarosegelen, die in der Küvettentechnik (8) hergestellt wurden, modifiziert. Mit diesen dünnen Gelen erhält 'man bessere Trennungen der Isoenzyme als mit der Originalmethode und vollkommen glatte Oberflächen nach dem Trocknen. Im übrigen wurde nach den in der Originalmethode gemachten Angaben gearbeitet.

\section{Ergebnisse}

\section{Enzymmuster}

Mit der Lektin-Affinitäts-Elektrophorese werden Leber-, Gallengangs- und Knochen-Isoenzym der alkalischen Phosphatase sehr guit voneinander getrennt. Die Qualität der Trennung hängt allerdings von der Lektinkonzentration im Gel ab. Bei der von Onica et al. (4) angegebenen niedrigen Lektin-Konzentration von $0,05 \mathrm{~g} / \mathrm{im} \mathrm{Gel}$ wird das Knochen-Isoenzym nur partiell präzipitiert, so daß regelmäßig eine zweite Knochenbande zwischen Leberund Gallengangs-Isoenzym zu sehen ist (Abb. 1, oberes Gel, Bahn 2, 4, 7). Die Knochenbande kann auch unvollständig von der Leberbande getrenint sein (Abb. 1, oberes Gel, Bahn 10). Bei einer Lektin-Konzentration von $0,4 \mathrm{~g} / 1$ im Gel, wie sie Schreiber und Whitta vorschlagen, bildet das Knochen-lsoenzym eine $M$-förmige Bande mit im allgemeinen nur geringer elektrophoretischer Wanderung. In Abhän'gigkeit von der Probe und der Aktivität des Knochen-Isoenzyms kann die elektrophoretische Wanderung sehr unterschiedlich sein (Abb. 1, 2). Vor der Bande kann sich ein Schweif bilden (Abb. 1, unteres Gel, Bahn 4). Die Schweifbildung ist besonders intensiv, wenn das Serum bei hoher Aktivität des Knochen-Isoenzyms mit 0,9\%iger Kochsalzlösung verdünnt wurde (Abb. 3, Bahn 8). Sie kann durch Verdünnen mit inaktiviertem Normalserum reduziert werden. Das Knochen-Isoenzym wandert dann allerdings weiter anodisch (Abb. 3, Bahn 9).

Außer dem Leber-, dem Gallengangs- und dem KnochenIsoenzym der alkalischen Phosphatase können mit der

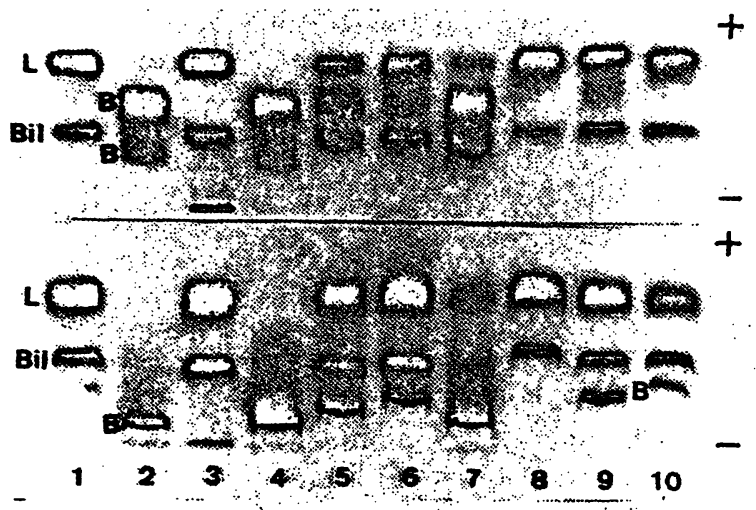

Abb. 1: Lektin-Affinitäts-Elektrophorese der alkalischen Phosphatase Isoenzyme bei unterschiedlichen Lektinkonzentrationen. Oberes Gel: 0,05 g/l. Unteres Gel: 0,4 g/l. Beide Gele enthalten $20 \mathrm{ml} / \mathrm{I}$ Triton $X-100$ (surfactant solution). $L=$ Leber-Isoenzym, $B i l=$ Gallengangs-Isoenzym, $B=$ Knochen-Isoenzym.

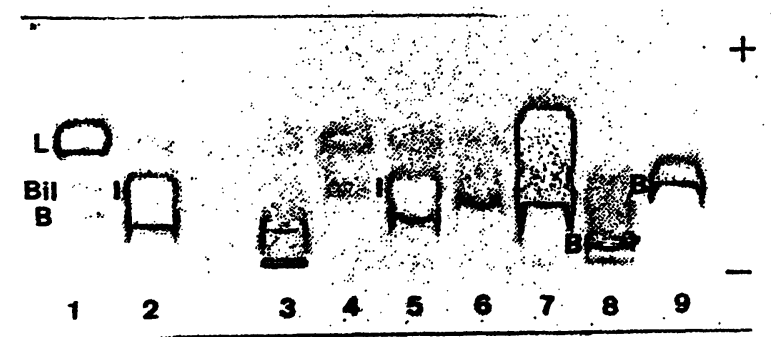

Abb. 3: Unterschiedliche Wanderung des Knochen-Isoenzyms der alkalischen Phosphatase in Abhängigkeit vom Verdünnungs-. medium. Bei Verdünnung mit inaktiviertem Normalserum (Bahn 9) wandert das Knochen-Isoenzym wesentlich weiter zur Anode als bei Verdünnung mit 0,9\%iger Kochsalzlösung (Bahn 8). Bei Verdünnung mit 0,9\%iger Kochsalzlösung (Bahn 8) ausgeprägte Schweifbildung infolge inkompletter Präzipitation des KnochenIsoenzyms durch Lektin.

\section{. 西

(1)

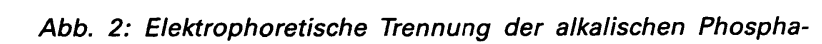
tase Isoenzyme in lektinhaltigem Gel (oben) und in lektinfreiem $\mathrm{Gel}$ (unten). Lektinkonzentration im oberen $\mathrm{Gel} 0,4 \mathrm{~g} / \mathrm{l}$. Beide Gele enthalten $20 \mathrm{ml} / \mathrm{I}$ Triton X-100 (surfactant solution). $L=L e-$ ber-Isoenzym, Bil = Gallengangs-Isoenzym, I = Dünndarm-Isoenzym, $B=$ Knochen-Isoenzym.

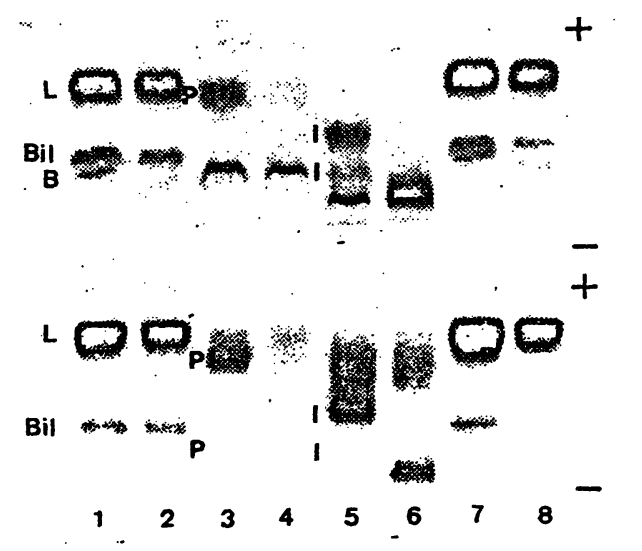

Abb. 4: Identifizierung von Placenta(P)- und Dünndarm(I)-Isoenzym der alkalischen Phosphatase. Das Placenta(P)-Isoenzym der alkalischen Phosphatase wandert im lektinhaltigen Gel (oben) etwas weniger anodisch als das Leber-Isoenzym. Im lektinfreien Gel (unten) hat es zwei charakteristische Banden (Bahn 3). Sowohl das Placenta(P)- als auch das Dünndarm(I)-Isoenzym werden durch den Antikörper gegen Placenta inhibiert (Bahn 4, 6). 

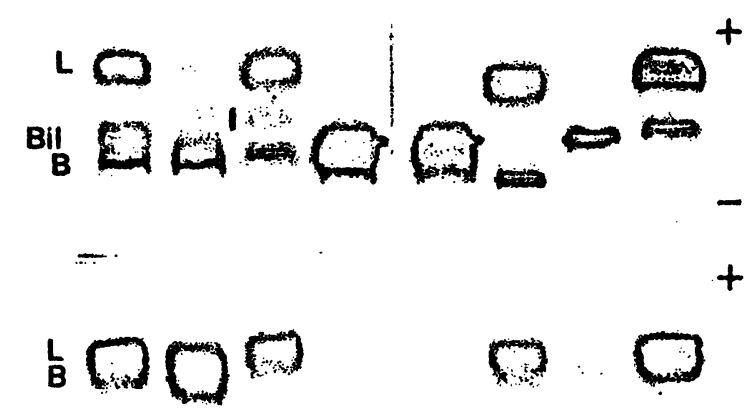

Bil

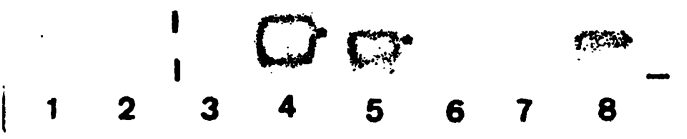

Abb. 5: Makro-Form der alkalischen Phosphatase (Bahn 4, 5). Typisch ist die kathodisch liegende Bande (*). Nach Spaltung des Enzym-Immunglobulinkomplexes mit Ficin sind die freien Isoenzyme nachweisbar, in diesem Fall Leber- und Knochen-Isoenzym (Bahn 6).

Lektin-Affinitäts-Elektrophorese auch das Dünndarm-Isoenzym (Abb. 2, Bahn 2, Abb. 4, Bahn 5) und das PlacentaIsoenzym der alkalischen Phosphatase (Abb. 4, Bahn 3) identifiziert werden. Es hat sich als nützlich erwiesen, jede Probe sowohl in einem lektinhaltigen als auch in einem lektinfreien Agarosegel zu trennen. Beide Gele enthalten Triton X-100 (surfactant solution), das die Präzipitation des Gallengangs-Isoenzyms durch Lektin verhindert. Mit dieser Kombination sind das Dünndarm- und das Placenta-Isoenzym eindeutig zu identifizieren (Abb. 4). AuBerdem kann die Verwechslung eines stark anodisch wandernden Knochen-Isoenzyms mit dem Gallengangs-Isoenzym vermieden werden (Abb. 2, Bahn 8, 9).

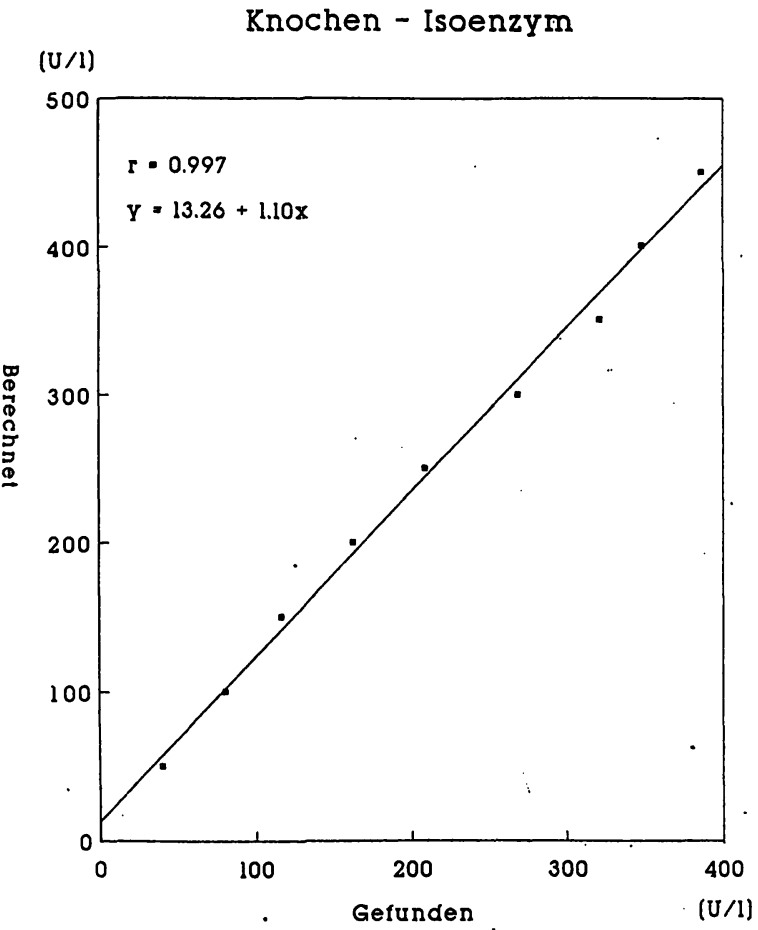

Abb. 7: Korrelation der gemessenen und der berechneten Knochen-AP-Aktivitäten in Serumgemischen.
$10000000000+$

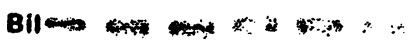

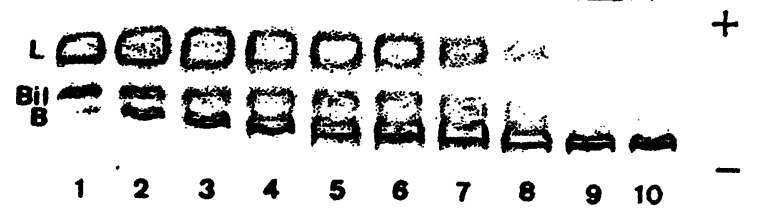

Abb. 6: Lektin-Affinitäts-Elektrophorese von Serumgemischen mit unterschiedlicher Aktivität des Leber-, Gallengangs- und Knochen-Isoenzyms der alkalischen Phosphatase. Die Regressionsgerade der berechneten und der gefundenen Werte für das Knochen-Isoenzym ist in Abbildung 7 dargestellt.

Der Verdacht auf das Vorliegen einer Makroform der alkalischen Phosphatase (immunglobulingebundene alkalische Phosphatase) ergibt sich, wenn im lektinfreien Gel eine isolierte, kathodennahe Isoenzymbande nachweisbar ist. Nach Spaltung des Enzym-Immunglobulinkomplexes mit der Protease Ficin stellt sich das freie Isoenzym dar (Abb. 5).

\section{Wiederfindung}

Ein Serum, das nur Leber- und Gallengangs-Isoenzym der alkalischen Phosphatase enthielt, wurde mit einem Serum, in dem nur das Knochen-Isoenzym nachweisbar

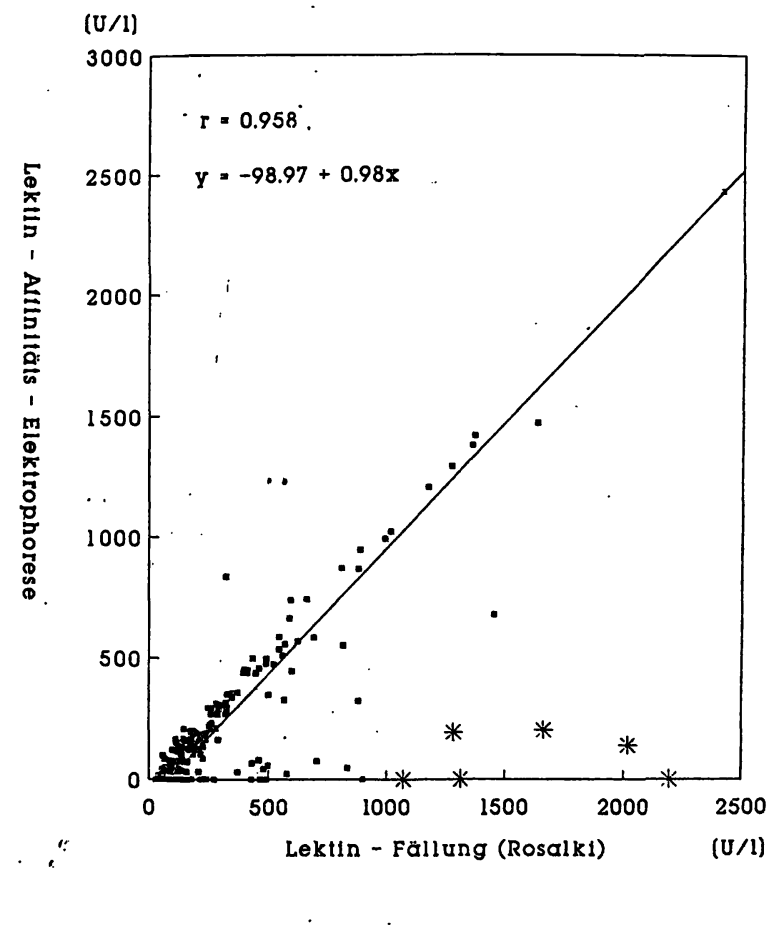

Abb. 8: Graphische Darstellung des Methodenvergleichs. Knochen-AP, gemessen mit der Lektin-Affinitäts-Elektrophorese und der Lektin-Fällungs-Methode von Rosalki. * Ausreißer gemäß (10). 


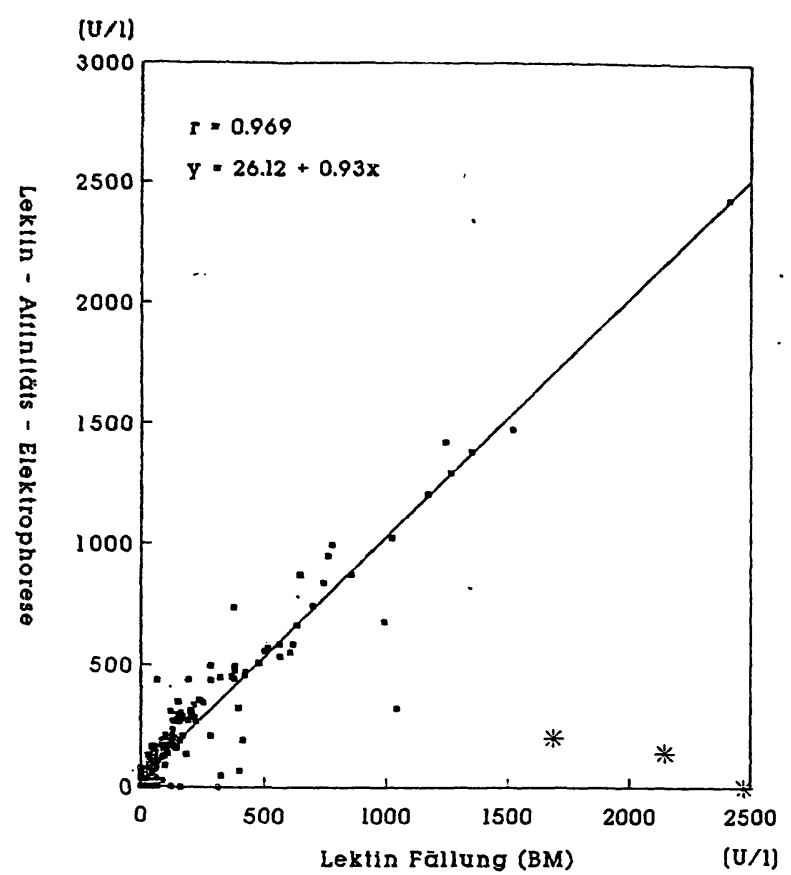

Abb. 9: Graphische Darstellung des Methodenvergleichs. Knochen-AP, gemessen mit der Lektin-Affinitäts-Elektrophorese und der Lektin-Fällungs-Methode von Boehringer Mannheim. * Ausreißer gemäß (10).

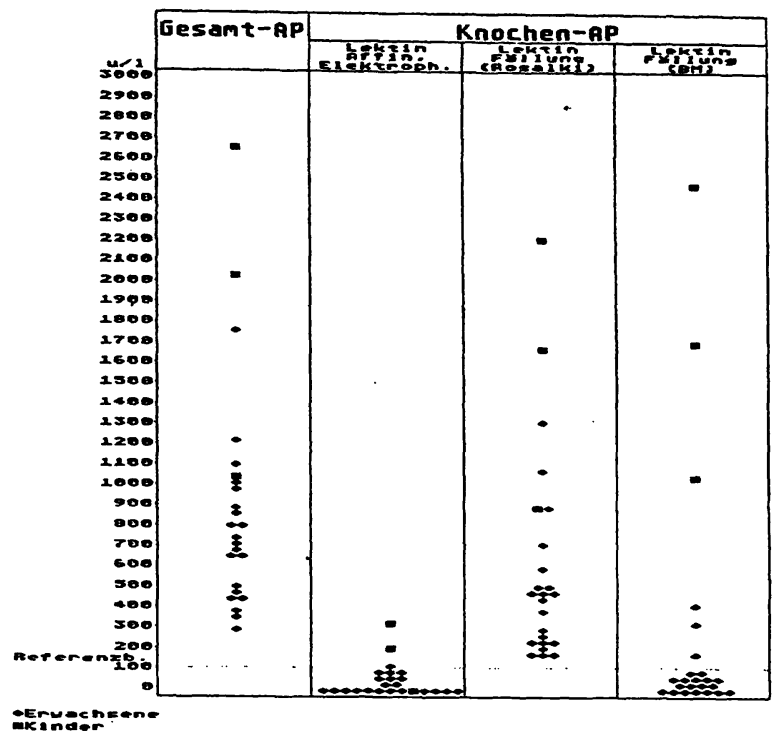

Abb. 10: Gesamt-AP und Knochen-AP von 24 Seren. In allen 24 Seren fehlte elektrophoretisch die Knochen-AP oder lag im Referenzbereich, während mit der Rosalki-Methode in allen 24 Seren und mit der Boehringer-Mannheim-Methode in 6 Seren deutlich erhöhte Aktivitäten der Knochen-AP gefunden wurden. Referenzwert der Knochen-AP für Kinder: < 544 U/I (3).

\section{Vergleich mit anderen Methoden}

In 200 Seren wurde das Knochen-Isoenzym der alkalischen Phosphatase mit der Lektin-Affinitäts-Elektrophorese und den Lektin-Fällungs-Methoden von Rosalki und Boehringer Mannheim gemessen. Die statistischen Auswertungen sind in Abb. 8 und 9 dargestellt. Die Ausgleichsgerade wurde als standardisierte Hauptkomponente nach Elimination der Ausreißer berechnet $(9,10)$.

Trotz der guten Korrelation zwischen der Lektin-AffinitätsElektrophorese und den Lektin-Fällungs-Methoden weisen in einer relativ großen Probenzahl die Isoenzym-Muster der alkalischen Phosphatase erhebliche Diskrepanzen auf.

Von den 200 untersuchten Seren fand sich in 122 Seren elektrophoretisch eine erhöhte Knochen-AP. Von diesen 122 Seren wiesen 3 Seren mit der Lektin-Fällung nach Rosalki und 27 Seren mit der Leḱtin-Fällung von Boehringer Mannheim normale Aktivitäten des Knochen-Isoenzyms

Tab. 1: Vergleich der Knochen-AP-Aktivitäten, die mit der LektinAffinitäts-Elektrophorese und den Lektin-Fällungs-Methoden gemessen wurden, in bezug auf den Referenzwert. $>$ Knochen-AP über dem Referenzwert. < Knochen-AP unterhalb des Referenzwertes. Die Zahlen der Seren mit nicht übereinstimmenden Befunden sind durch Umrandung hervorgehoben.

\begin{tabular}{|c|c|c|c|c|}
\hline \multirow{2}{*}{$\begin{array}{l}\text { Lektin-Affinitäts- } \\
\text { Elektrophorese } \\
\text { n }\end{array}$} & \multicolumn{4}{|c|}{$\begin{array}{l}\text { Lektin-Fällungs-Methoden } \\
\text { Rosalki Boehringer-Mannheim }\end{array}$} \\
\hline & $>$ & $<$ & $>$ & $<$ \\
\hline 122 & 119 & 3 & 95. & 27 \\
\hline 78 & 46 & 32 & 国 & 72 \\
\hline
\end{tabular}

\section{Referenzwerte}

Für die Ermittlung der Referenzwerte wurden je 20 Seren von klinisch gesunden Frauen und Männern mit normalen Transaminasen und normaler $y$-Glutamyl-Transpeptidase untersucht. Die folgenden vorläufigen Referenzwerte wurden ermittelt (90\% Vertrauensbereich).

Leber-Isoenzym: Frauen < 90 U/I, Männer < $100 \mathrm{U} / \mathrm{l}$. Gallengangs-Isoenzym: Frauen < $10 \mathrm{U} / \mathrm{l}$, Männer < $10 \mathrm{U} / \mathrm{l}$. Knochen-Isoenzym: Frauen < 90 U/I, Mạnner < $100 \mathrm{U} / \mathrm{l}$. Dünndarm-Isoenzym: Frauen < $10 \mathrm{U} / \mathrm{l}$, Männer < $10 \mathrm{U} / \mathrm{l}$. 


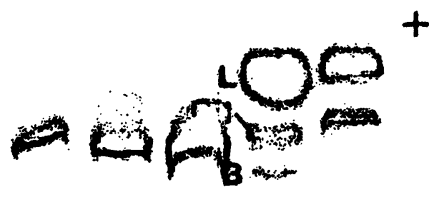

$\begin{array}{lllll}1 & 2 & 3 & 4 & 5\end{array}$

Tab. 2: Gesamt-Aktivität der alkalischen Phosphatase und Aktivität der Knochen-AP, gemessen mit den beiden Lektin-FällungsMethoden und mit der Lektin-Affinitäts-Elektrophorese bei $10 \mathrm{~Pa}$ tienten mit bekannter Diagnose. $L=$ Leber-, $G=$ Gallengangs$K=$ Knochen-Isoenzym der alkalischen Phosphatase. Patient 5 ist ein Kind. Referenzwert der Knochen-AP für Kinder: < 544 U/I (3). Das elektrophoretische Isoenzymmuster dieses Patienten findet sich in Abbildung 11 (Bahn 4)

\begin{tabular}{|c|c|c|c|c|c|c|}
\hline Patient & $\begin{array}{l}\text { AP } \\
\text { ges. } \\
\text { (U/I) }\end{array}$ & $\begin{array}{r}\text { Kno } \\
\text { Rosalki }\end{array}$ & $\begin{array}{l}\text { Ochen-AP } \\
\text { Boehringe } \\
\text { Mannheim }\end{array}$ & $\begin{array}{l}\text { U/I) } \\
\text { Elektro- } \\
\text { phorese }\end{array}$ & $\begin{array}{l}\text { Elektro- } \\
\text { phore- } \\
\text { tisches } \\
\text { Isoenzym- } \\
\text { Muster }\end{array}$ & Diagnose \\
\hline 1 & 505 & 235 & 32 & 0 & UG & $\begin{array}{l}\text { Choledochus- } \\
\text { stenose }\end{array}$ \\
\hline 2 & 645 & 429 & 401 & 67 & $U G /(K)$ & $\begin{array}{l}\text { Cystische Pan- } \\
\text { kreasfibrose }\end{array}$ \\
\hline 3 & 2654 & 2194 & 2469 & 0 & LG & $\begin{array}{l}\text { Gallengangs- } \\
\text { atresie }\end{array}$ \\
\hline 4 & 689 & 369 & 7 & 30 & $U G /(K)$ & $\begin{array}{l}\text { Pankreaskopf- } \\
\text { carcinom mit } \\
\text { Cholestase }\end{array}$ \\
\hline 5 & 2230 & 1662 & 1686 & 202 & $U G /(K)$ & $\begin{array}{l}\text { Cystische Pan- } \\
\text { kreasfibrose }\end{array}$ \\
\hline 6 & 730 & 466 & 52 & 0 & LG & Lebercirrhose \\
\hline 7 & 699 & 427 & 30 & 0 & L/G & $\begin{array}{l}\text { Leberstauung } \\
\text { bei Mitralinsuf- } \\
\text { fizienz }\end{array}$ \\
\hline 8 & 874 & 490 & 0 & 0 & LG & $\begin{array}{l}\text { Lebermetasta- } \\
\text { sen bei Dick- } \\
\text { darmcarcinom }\end{array}$ \\
\hline $\begin{array}{r}9 \\
10\end{array}$ & $\begin{array}{r}1218 \\
287\end{array}$ & $\begin{array}{l}834 \\
271\end{array}$ & $\begin{array}{r}326 \\
0\end{array}$ & $\begin{array}{r}47 \\
0\end{array}$ & $\begin{array}{c}U G /(K) \\
\text { UG }\end{array}$ & $\begin{array}{l}\text { Cholelithiasis } \\
\text { Lebermetasta- } \\
\text { sen }\end{array}$ \\
\hline
\end{tabular}

Abb. 11: Typisches Leber/Gallengangs-Isoenzym-Muster mit nur schwacher Knochen-Isoenzym-Bande bei einem Kind mit Mucoviscidose (Bahn 4). Mit beiden Lektin-Fällungs-Methoden war dagegen überwiegend das Knochen-Isoenzym der alkalischen Phosphatase nachweisbar (Patient 5, Tab. 2).

auf. 78 der 200 untersuchten Seren hatten in der LektinAffinitäts-Elektrophorese eine Aktivität der Knochen-AP im Referenzbereich. In 46 Seren dieser Gruppe war das Knochen-Isoenzym der alkalischen Phosphatase mit der Methode von Rosalki und in 6 Seren mit der BoehringerMannheim-Methode deutlich erhöht. Alle diese Seren hatten elektrophoretisch ein typisches Leber-Gallengangs-Isoenzymmuster. Das Knochen-Isoenzym fehlte entweder ganz oder war nur in Spuren vorhanden. Die Ergebnisse sind in Tabelle 1 zusammengefaßt.

24 dieser Seren wiesen besonders diskrepante IsoenzymMuster auf. Obwohl elektrophoretisch das Knochen-Isoenzym entweder ganz fehlte oder im Referenzbereich lag, fanden sich inallen Seren mit der Rosalki-Methode und in 6 Seren mit dem Fällungsreagenz von Boehringer Mannheim überwiegend Knochen-AP. Die Verhältnisse sind graphisch in Abbildung 10 dargestellt. Bei 10 Patienten dieser Gruppe konnten klinische Informationen erhalten werden. Die in Tab. 2 aufgeführten Diagnosen sprechen für die Richtigkeit der elektrophoretisch gefundenen Isoenzym-Muster (Abb. 11). Bei keinem der 10 Patienten ergaben sich klinische Hinweise auf eine Knochenerkrankung. Mit den Lektin-Fällungs-Methoden wird offensichtlich in einem Teil der Seren durch unspezifische Fällung das Vorliegen von Knochen-AP vorgetäuscht.

\section{Diskussion}

Mit der elektrophoretischen Trennung in lektinhaltigen und lektinfreien dünnschichtigen Agarosegelen können die Isoenzyme der alkalischen Phosphatase (Leber-, Gallengangs-, Knochen-, Dünndarm-, Placenta- und MakroAP) zuverlässig getrennt und quantitativ bestimmt werden. Die elektrophoretisch gemessenen Aktivitäten des Knochen-Isoenzyms korrelieren gut mit den Aktivitäten, die mit den beiden Lektin-Fällungs-Methoden bestimmt wurden. Dennoch gibt es in relativ vielen Seren erhebliche Diskrepanzen zwischen der Lektin-Affinitäts-Elektrophorese und den Lektin-Fällungs-Methoden. Besonders mit der Rosalki-Methode wird in einigen Seren alkalische Phosphatase unspezifisch gefällt und dadurch eine erhöhte Knochen-AP vorgetäuscht. Dieses Phänomen tritt bei der Boehringer-Mannheim-Methode wesentlich seltener auf. Falsch erhöhte Aktivitäten des Knochen-Isoenzyms der alkalischen Phosphatase finden auch Behr et al. mit der Lektin-Fällungs-Methode (3).

Elektrophoretisch können auch seltener vorkommende Isoenzyme der alkalischen Phosphatase wie Dünndarm-,

Placenta und Makro-AP' getrennt und quantitativ bestímmt werden. Diese Isoenzyme werden mit Lektin nicht gefällt. Sie sind ein Teil der Restaktivität. Schwerwiegende Fehldiagnosen sind möglich, wenn diese selteneren Isoenzyme in hoher Aktivität im Überstand nach Lektin-Fällung vorhanden sind und die Restaktivität als Leberisoenzym fehlgedeutet wird.

Aufgrund der hier mitgeteilten Ergebnisse können die Lektin-Fällungs-Methoden zur Messung der Knochen-AP nur mit Vorbehalt empfohlen werden. Wegen ihrer im Vergleich zur Elektrophorese höheren Präzision könnten sie sich jedoch für Verlaufskontrollen eignen. Allerdings müßte wenigstens einmal das Isoenzym-Muster der alkalischen Phosphatase elektrophoretisch untersucht werden. Für die Abklärung einer klinisch nicht einzuordnenden erhöhten Aktivität der alkalischen Phosphatase müssen in jedem Fall qualifizierte elektrophoretische Verfahren eingesetzt werden, damit auch seltener vorkommende Isoenzyme zuverlässig erkannt und Fehldiagnosen vermieden werden.

Schriftum:

1. MEYER-SABELleK, W., SINHA, P., KOTTGEN, E.: Alkaline Phosphatase. Laboratory and clinical Implications. J. of Chromatography 429, 419-444 (1988).

2. ROSALKI, S. B., YING FOO, A.: Two new methods for separation and quantifying bone and liver alkaline phosphatase isoenzymes in plasma. Clin. Chem. 30, 1182-1186 (1984).

3. BEHR W. BARNERT, J.: Quantification of Bone Alkaline Phosphatase in Serum by Precipition with Wheat-Germ Lectin: A Simplified Method and Its Clinical Plausibility. Clin. Chem. 32, 1960-1966 (1986).

4. ONICA. D. SUNDBLAD, L., WALDENLIND, L.: Affinity electrophoresis of human 4. ONICA; D., SUNDBLA, ., WALENe is a a rose gel containing lectin. Clin. Chim. serum alkaline phosphatase isoenzymes in agarose gel containing lectin. Clin. Chim. Acta 155, 285-294 (1986)

5. SCHREIBER, W. E., WHITTA, L.: Alkaline Phosphatase Isoenzymes Resolved by Elec trophoresis on Lectin-Containing Agarose Gel. Clin. Chem. 32, 1570-1573 (1986). 
6. ROSALKI, S. B., YING FOO, A.: Lectin affinity electrophoresis of alkaline phosphatase for the differentiation of bone and hepatobiliary disease. Electrophoresis 10 , 604-611 (1989).

7. KLEIN, G., BODENMÜLLER, H., GERBER, M.: Evalution of a Now Assay for the Determination of Human Bone Alkaline Phosphatase. .8.th International Congress of Clinical Enzymology" May 30-June 1, 1989 Toronto, Canada.

8. N. N.: The SERVA Gel Casting Kit. Cat. No. 42938, SERVA FEIN BIO CHEMICA GmbH, 6900 Heidelberg.

9. HAECKEL, R.: Empfehlungen zur Anwendung statistischer Methoden beim Vergleich klinisch-chemischer Analysenverfahren. Bericht uber die Kleinkonferenz der Deutschen Gesellschaft für Klinische Chemie am 22. und 23. Juli 1981 in Hannover. J. Clin. Chem. Clin. Biochem. 20, 107-110 (1982).

10. CORNBLEET, P. J., GOCHMAN, N.: Incorrect Least-Squares Regression Coefficients in Method-Comparison. Clin. Chem. 25, 432-438 (1979).

11. N. N.: Isoenzyme der alkalischen Phosphatase. diagnostica dialog 2, 11 (1989).

\section{Danksagung}

Herrn Andreas Spiller und Herrn Wolf Spiller danke ich für die sorgfältige Durchführung der elektrophoretischen Trennungen. Herrn Jens Engler und Herrn Martin Scholze danke ich für die statistischen Berechnungen.

Anschrift des Verfassers:

Dr. med. Hans-Walter Schiwara

Straßburger Straße 19

2800 Bremen 1

\section{Mitteilung des Verbandes der Diagnostica- und Diagnosticageräte-Hersteller e.V. Stellungnahme des VDGH-Ausschusses "Immundiagnostica"}

zum Leserbrief der Herren Professoren Röhle und Siekmann zu „Ringversuche für Plasmaproteine, Hormone und Pharmaka auf Basis der neuen Qualitätssicherungsrichtlinien der Bundesärztekammer (Rili-BÄK)“ in Lab.med. 14: 360 (1990).

1. Vorab wird ausdrücklich begrüßt, daß die beiden Verfasser nunmehr in Form ihres Leserbriefes einige Grundsätze zu dem Thema publizieren. Absicht des VDGH und seines Ausschusses "Immundiagnostica" war es, mit dem Workshop vom 6. Oktober 1989 die Diskussion mit allen Beteiligten und Interessierten fortzuführen. Wir glauben, daß der Workshop hierzu einen vernünftigen Ansatz gebracht hat, sind uns allerdings bewußt, daß noch weitere Arbeiten und Diskussionen nötig sind und werden diesbezüglich auch erneut die Initiative ergreifen.

2. Der Beitrag ist nicht anonym an die Zeitschrift "Laboratoriumsmedizin" geschickt und von dieser auch nicht anonym veröffentlicht worden. Vielmehr geht aus den einleitenden Anmerkungen hervor, daß aufgrund der Tatsache, daß nur ein Teil der Referate zur Veröffentlichung den Veranstaltern des Workshops eingereicht wurde, der Ausschuß „Immundiagnostica“ des VDGH als Veranstalter eine Zusammenfassung der wesentlichen Aussagen versucht hat. Ziel war es, auch hiermit die Diskussion zu diesem wichtigen Thema voranzutreiben, was auch gelungen ist.

3. Der Leserbrief zeigt die Auffassung der Autoren, die ebenfalls in der Bundesärztekammer vertreten wird (siehe z. B. die Veröffentlichung "AWA" im Deutschen Ärzteblatt 87 [1990] Seite A-281) und die auch immer wieder von den Verfassern der "Rili-BÄK” zum Ausdruck gebracht wird, sehr deutlich, daß mit den "Rili-BÄK" eine Standardisierung erreicht werden soll, die über die Gewährleistung einer vernünftigen Vergleichbarkeit der Ergebnisse und einer den medizinischen Erfordernissen entsprechenden Aussagekraft hinausgeht. Diese Auffassung entspricht weder dem gesetzlichen Auftrag der Eichordnung noch dem Titel der "Rili-BÄK". Sie sollen nämlich der Qualitätssicherung in medizinischen Laboratorien dienen, d. h. die ärztliche Handlung, "Erhebung eines labormedizinischen Befundes", auf. ihre Richtigkeit überprüfen. So sagt $\S 4$ der Eichordnung, die Ermächtigungsnorm für den Erlaß der „Rili-BÄK”, daß derjenige, der mit medizinischen Meßgeräten quantitative labormedizinische Untersuchungen durchführt, die Meßergebnisse durch Kontrolluntersuchungen (laborinterne Qualitätskontrollen) und durch Teilnahme an jährlich zwei Vergleichsmessungen (Ringversuche) zu überwachen hat, nicht aber, daß er bzw. andere zur Standardisierung in obigem Sinne gezwungen werden sollen.

Es geht also ausschließlich um die Qualitätssicherung sprich Überwachung der Richtigkeit der Meßergebnisse - der "ärztlichen Leistung Laboratoriumsuntersuchung”.

4. Aus obiger Vermengung des Wunșches nach Standardisierung mit dem gesetzlichen Auftrag, Qualität zu kontrollieren, folgt ein weiteres Mißverständnis: Es geht nicht um eine analytische Genauigkeit an und für sich, sondern nur um eine Genauigkeit, die den medizinischen Erfordernissen entspricht. So führt Prof. Stamm in seinem Vorwort zur Veröffentlichung der "Rili-BÄK" im Deutschen Ärzteblatt 85 (1988) auf Seite A 697 aus:

„Die Vorgaben für die laborinterne Qualitätssicherung und die Ringversuche werden nicht so hoch wie technisch möglich angesetzt, sondern sie richten sich nach den medizinischen Erfordernissen. Die medizinischen Erfordernisse werden aufgrund der Auswirkungen der Vorgaben für die Qualitätskontrolle auf die medizinische Beurteilung eines Analysenergebnisses festgelegt."

5. Die Industrie hat nicht nur Verständnis für den Wunsch der Durchführer von Laboratoriumsuntersuchungen nach Standardisierung, sondern ist selbst maßgeblich an der Realisierung dieser Ziele beteiligt. Allerdings ist sie der Meinung, daß dies nicht im Rahmen der Qualitätssicherung im Labor - sei es auf Basis von "Rili-BÄK" oder etwaiger anderer Maßnahmen - erfolgen sollte, sondern durch die Erarbeitung von weltweit anerkannten Referenzmaterialien.

\subsection{Referenzmaterialien}

Seit vielen Jahren arbeiten die betroffenen Industriefirmen aktiv im Rahmen der WHO an der Erarbeitung von weltweit anerkannten Referenzmaterialien mit. Darüber hinaus hat sich gerade auch die im VDGH zusammengeschlossene Industrie spezieller Themen angenommen:

- Seit längerem arbeiten. Experten der Industrie unter Beteiligung von interessierten Wissenschaftlern aus dem Hochschulbereich an der Erarbeitung eines "ReferenzNormalplasmas zur ISI/INR-Kalibrierung". Dieses ist żwischenzeitlich hergestellt und die Sollwerte ermittelt wor- 